Gut, 1973, 14, 64-67

\title{
Ischaemic colitis and abdomino-perineal excision of the rectum
}

\author{
M. LEA THOMAS AND J. M. WELLWOOD \\ From the Departments of Radiology and Surgery, St Thomas' Hospital, London
}

SUMMARY Two patients are presented in whom ischaemic colitis followed some years after abdomino-perineal excision of the rectum for carcinoma. The first patient was a young man without evidence of arterial disease and the second patient suffered from auricular fibrillation, thought to be due to ischaemic heart disease.

Ligation of the inferior mesenteric artery in the operation of abdomino-perineal excision of the rectum may reduce the blood flow through the marginal artery of Drummond rendering the remaining colon more liable to ischaemic damage.

Patients who pass bright blood through a colostomy following abdomino-perineal excision of the rectum for carcinoma may have ischaemic colitis rather than a recurrence of the neoplasm.

The term ischaemic colitis was first used by Marston, Pheils, Lea Thomas, and Morson (1966) to describe a form of acute colitis presenting with abdominal pain and bloodstained diarrhoea in which there was clinical, radiological, and pathological evidence of ischaemia. The lesion frequently involves the splenic flexure, and in a series reported by one of us (Thomas, 1972) this part of the colon was involved in $80 \%$ of patients. The reason for this predilection for the region of the splenic flexure may be explained on anatomical grounds. The arterial supply to the colon is by the vasa recti arising from the marginal artery of Drummond. The marginal artery is formed by anastomosis between branches of the superior mesenteric artery and by the left colic and sigmoid branches of the inferior mesenteric artery. The anastomosis between the inferior and superior mesenteric arteries through the marginal artery is generally well developed and has been demonstrated experimentally (Griffiths, 1956). At the splenic flexure, however, the marginal artery is poorly developed, but is augmented by the two ascending branches of the left colic artery. If the left colic artery is occluded either by disease or by surgical intervention, as in the operation of abdominoperineal excision of the rectum with high ligation of the inferior mesenteric artery, this part of the colon may become ischaemic (Griffiths, 1956; Griffiths, 1966).

Of 19 patients with ischaemic colitis seen at this Received for publication 16 November 1972. hospital between the years 1961 and 1971, two had previously undergone abdomino-perineal excision of the rectum for carcinoma.

This paper describes the clinical and radiological features of these two patients.

\section{Case Reports}

CASE 1

A man, aged 43, underwent abdomino-perineal excision of the rectum for carcinoma. The level at which the inferior mesenteric artery was ligated was not recorded. The patient remained well without evidence of recurrence of the tumour. Four years after the operation he was admitted complaining of colicky abdominal pain, vomiting, and loose motions. He was tender in the lower abdomen and digital examination of the colostomy revealed bloody fluid within the lumen. There were no other abnormal signs and in particular there was no evidence of vascular disease. Shortly after admission he passed large quantities of fresh blood per colostomy.

At sigmoidoscopy a cobblestone appearance of the mucosa with a stenosis between 15 and $21 \mathrm{~cm}$ from the colostomy site was observed. Above $21 \mathrm{~cm}$ the mucosa appeared normal.

A barium enema showed irregular narrowing and 'thumbprinting' which extended from the colostomy as far as the splenic flexure (Fig. 1a). The patient's symptoms subsided and another barium enema two months later showed return to normal (Fig. 1b). He 


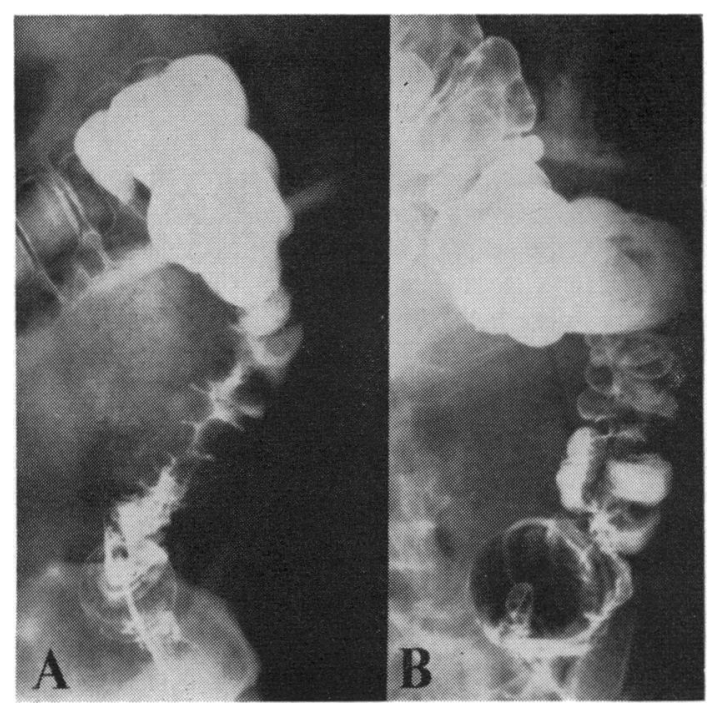

Fig. 1 (case 1) A Barium enema three days after onset of symptoms. There is narrowing and 'thumbprinting' extending from the colostomy to the splenic flexure.

B Repeat barium enema two months later. The affected colon is now normal.

remained asymptomatic for two more years and was then readmitted with a further episode of vomiting, abdominal pain, and passage of fresh blood per colostomy. The barium enema demonstrated a 10$\mathrm{cm}$ long narrowing at the splenic flexure with 'thumbprinting' (Fig. 2a). The symptoms again subsided spontaneously, and a further barium enema a week

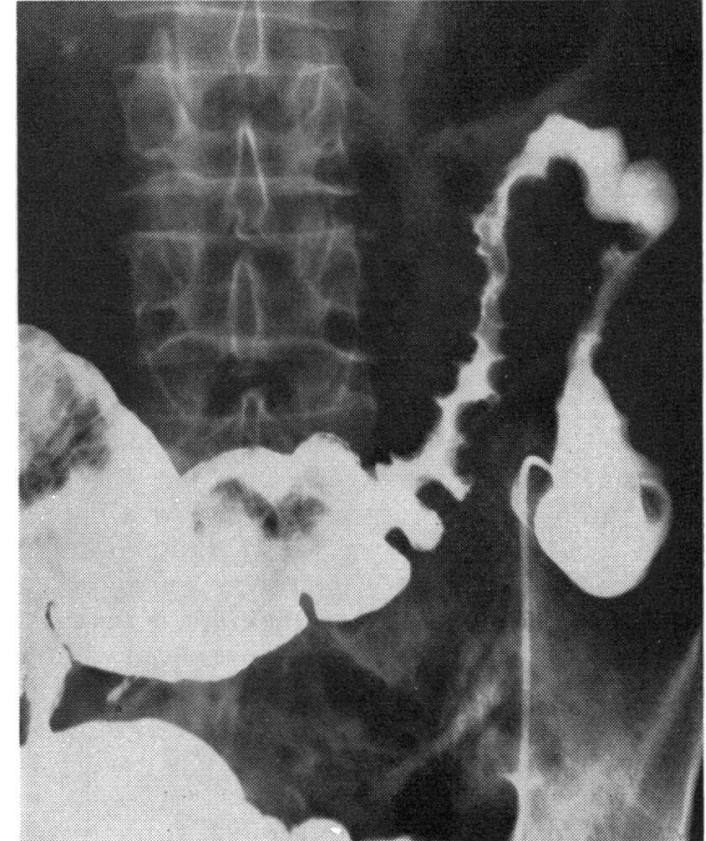

Fig. 3 (case 2) Barium enema five days after onset of symptoms. There is narrowing and 'thumbprinting' of the left third of the transverse colon, the splenic flexure and descending colon as far as the colostomy.

later showed persistent narrowing at the splenic flexure (Fig. 2b). A third barium enema six weeks after the onset of symptoms showed only a mild residual narrowing at the splenic flexure (Fig. 2c).

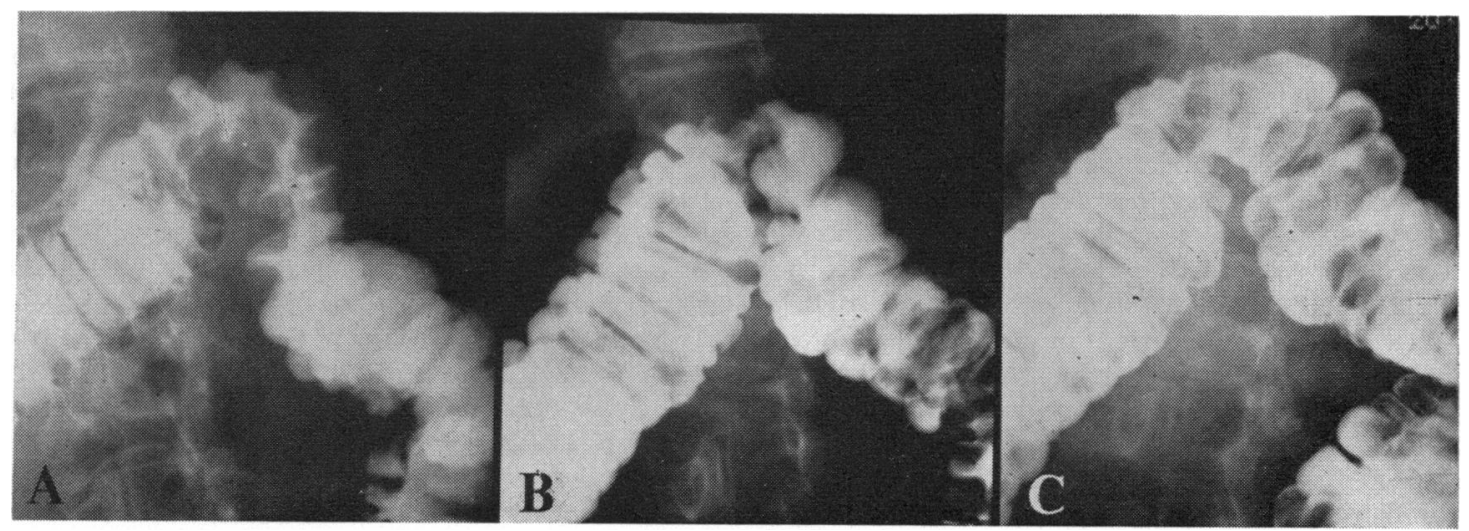

Fig. 2 (case 1) A Barium enema two days after the second admission. There is narrowing and 'thumbprinting' at the splenic flexure.

B Repeat barium enema one week later. There is persistent narrowing at the splenic flexure.

C Barium enema six weeks after the onset of symptoms. Only a mild stricture at the splenic flexure remains. 
The patient has since remained asymptomatic and there are no signs of recurrent tumour.

\section{CASE 2}

A 60-year-old man underwent an abdomino-perineal excision of the rectum for carcinoma in 1966. At the time of this admission he suffered from atrial fibrillation and an attempt to reverse this by cardioversion and digitalization was unsuccessful. The cause of the auricular fibrillation was thought to be ischaemic heart disease.

Two and a half years after the operation the patient complained of the passage of bright blood per colostomy. A recurrent carcinoma or a second primary tumour was suspected. However a barium enema showed narrowing and 'thumbprinting' involving the left third of the transverse colon, the splenic flexure, and the descending colon as far as the colostomy (Fig. 3). The symptoms resolved without treatment and he has remained symptom free without signs of recurrent tumour for three years.

\section{Discussion}

The clinical and radiological signs of ischaemic colitis have been described by one of us in detail elsewhere (Marson et al, 1966; Thomas, 1972). In both patients discussed here the clinical and radiological signs were typical of the condition, as was the spontaneous recovery of both patients without specific treatment.

Although a causal relationship between the previous abdomino-perineal excision of the rectum and the development of ischaemic colitis cannot be proved, these two patients constitute $10 \%$ of patients with ischaemic colitis seen at this hospital in the last decade.

As the ischaemic colitis did not follow immediately upon excision of the rectum in either patient, the anastomotic pathways through the marginal arteries at the time of operation must have been adequate to maintain the blood supply to the left side of the colon. It seems likely, however, that ligation of the inferior mesenteric artery played a role in the subsequent development of ischaemic colitis, a view supported by the fact that in both these patients ischaemic colitis occurred in the territory of the inferior mesenteric artery, which, although a common site, is by no means the only one (Farman, 1971). After excision of the rectum, reduction of blood flow to the marginal artery supplying the residual colon would be most marked if the ligation of the inferior mesenteric artery had been performed flush to the aorta and therefore proximal to the origin of the left colic artery, or if the left colic artery itself had been ligated.
Goligher (1954), observing the effect of a high ligation of the inferior mesenteric artery at operation, concluded that in the majority of patients the remaining blood supply to the colon and rectum was adequate, and Griffiths (1956) states that in the absence of arterial disease or vascular anatomical anomaly, the distal colon and rectum may be adequately vascularized by the branches of the superior mesenteric artery through the marginal artery. Out of 220 patients who underwent excision of the distal colon or rectum, with ligation of the inferior mesenteric artery at its origin, Morgan and Griffiths (1959) record one patient with necrosis of a left iliac colostomy, and another patient whose death following operation may have been attributable to devascularization of the colon. Marston (personal communication), in a review of 122 cases of ischaemic colitis, describes one patient who developed ischaemic colitis following abdomino-perineal excision of the rectum.

Our first patient was a young man with no evidence of arterial disease. One possible aetiological factor could have been the unrecognized presence of a vascular anomaly in the colonic blood supply. The middle colic artery was absent in $22 \%$ of specimens studied by Griffiths (1956) and the absence of this vessel or the existence of other arterial anomalies may result in ischaemia of the distal colon should the inferior mesenteric artery be ligated or occluded (Harrison and Croal, 1962). At operation it may be difficult to assess the state of the visceral vessels or the presence of anatomical abnormalities, and flush ligation of the inferior mesenteric artery during excision of the colon or rectum has been followed by necrosis of part or all the remaining large bowel in the postoperative period (Shaw and Green, 1953; Bernstein and Bernstein, 1963). However, if a congenital abnormality were the cause, ischaemia would be expected to occur shortly after operation, and the four-year interval between operation and the development of ischaemic colitis is difficult to explain.

The second patient in our series had suffered from persistent atrial fibrillation and it has been recognized that ischaemic colitis may be associated with heart disease, with or without auricular fibrillation, the latter giving rise to emboli (Marston et al, 1966; Thomas, 1968). In this patient, recurrent small emboli may have further compromised the previously reduced marginal arterial supply resulting in an episode of ischaemic colitis. Alternatively, as this patient was considered to have ischaemic heart disease, generalized arteriosclerosis may have gradually reduced the collateral supply to the left side of the colon resulting in ischaemia two years after ligation of the inferior mesenteric artery. As angiography was not carried out in either of our 
patients, the precise arterial lesion, if any, is not known.

Venous occlusion unrelated to the ligation of the inferior mesenteric artery could have caused the ischaemic colitis. Clinically, radiologically, and experimentally it is impossible to distinguish with certainty ischaemic colitis due to venous as opposed to arterial occlusion (Marcuson and Farman, 1971; Marcuson, Stewart, and Marston, 1972). The presence of abdominal neoplasm is known to predispose to venous thrombosis. However there has been no evidence of spread of the rectal carcinoma in either patient.

Ischaemic colitis should be considered in all patients who had undergone surgical ligation of the inferior mesenteric artery and subsequently develop abdominal pain and passage of bright red blood per colostomy or rectally. Barium studies should be carried out as soon as the diagnosis is suspected otherwise the chance to demonstrate an ischaemic lesion may be missed.

\section{References}

Bernstein, W. C., and Bernstein, E. F. (1963). Ischaemic ulcerative colitis following inferior mesenteric arterial ligation. Dis. Colon Rect., 6, 54-61.

Farman, J. (1971). The radiologic features of colonic vascular disease. In Vascular Disorders of the Intestine, edited by Scott J. Boley, S. S. Schwartz, and L. F. Williams, Jr., pp, 229-241. Butterworths, London. Appleton-Century-Crofts, New York.

Goligher, J. C. (1954). The adequacy of the marginal blood supply to the left colon after high ligation of the inferior mesenteric artery during excision of the rectum. Brit. J. Surg., 41, 351-353.

Griffiths, J. D. (1956). Surgical anatomy of the blood supply of the distal colon. Ann. Roy. Coll. Surg. Engl., 19, 241-256.

Griffiths, J. D. (1966). The blood supply of the colon, Proc. roy. Soc. Med., 59, 881-882.

Harrison, A. W., and Croal, A. E. (1962). Left colon ischemia following occlusion or ligation of the inferior mesenteric artery. Canad. J. Surg., 5, 293-298.

Marcuson, R. W., and Farman, J. A. (1971). Ischaemic disease of the colon. Proc. roy. Soc. Med., 64, 1080-1083.

Marcuson, R. W., Stewart, J. O., and Marston, A. (1972). Experimental venous lesions of the colon. Gut, 13, 1-7.

Marston, A. (1972). Personal communication.

Marston, A., Pheils, M. T., Lea Thomas, M., and Morson, B. C. (1966). Ischaemic colitis. Gut, 7, 1-15.

Morgan, C. N., and Griffiths, J. D. (1959). High ligation of the inferior mesenteric artery during operations for carcinoma of the distal colon and rectum. Surg. Gynec. Obstet., 108, 641-650.

Shaw, R. S., and Green, T. H., Jr. (1953). Massive mesenteric infarction following inferior mesenteric-artery ligation in resection of the colon for carcinoma. New Engl. J. Med., 248, 890-891.

Thomas, M. L. (1968). Further; observations on ischaemic colitis. Proc. roy. Soc. Med., 61, 341-342.

Thomas, M. L. (1972). Radiology-plain films and barium studies of the ischaemic bowel. Clin. Gastroent., 1, 581-595. 